\title{
A rare case of a gigantic myxoid liposarcoma of vulva: case report
}

\author{
Tanudeep Kaur ${ }^{1 *}$, Ravinder P. Singh ${ }^{2}$
}

${ }^{1}$ Department of Gynaecology, Division of Urogynaecology, AIIMS, New Delhi, India

${ }^{2}$ Centre for Oncosurgery, Saroj Super Speciality Hospital, Delhi, India

Received: 05 January 2021

Accepted: 10 February 2021

*Correspondence:

Dr. Tanudeep Kaur,

E-mail: tanibedi@gmail.com

Copyright: $\odot$ the author(s), publisher and licensee Medip Academy. This is an open-access article distributed under the terms of the Creative Commons Attribution Non-Commercial License, which permits unrestricted non-commercial use, distribution, and reproduction in any medium, provided the original work is properly cited.

\begin{abstract}
Vulval malignancies per se are very rare and a liposarcoma in this location is rarer still. The literature consists only of case reports of patients, often with a very limited follow up. We present a rare case of a young 30 year old unmarried nulliparous woman presenting with a giant vulval mass of $30 \times 20 \mathrm{~cm}$ and weighing nearly 6 kilograms. Ultrasonography, Computed Tomography, Magnetic Resonance Imaging and biopsy were done. Local resection with adjuvant radiotherapy was given. Histopathology was suggestive of myxoid liposarcoma and the patient is presently recurrence free with over 8 years of follow up. Though rare, myxoid liposarcoma should be kept in differential diagnosis of vulval soft tissue mass. Management includes a combination of surgery and radiation. Excision of lymph nodes is not recommended. Strict prolonged follow up for recurrence or metastasis is mandatory, and any new complaints should be addressed promptly. This is particularly true in the setting of liposarcoma, which may exhibit unusual patterns of spread and recurrence.
\end{abstract}

Keywords: Myxoid liposarcoma, Vulvar mass, Vulvar tumor, Giant mass, Case report

\section{INTRODUCTION}

Vulval malignancies constitute only $5 \%$ of total gynaecological malignancies with sarcomas being particularly rare (1-3\% of vulval malignancies or $0.05-$ $0.15 \%$ of total gynaecological malignancies). ${ }^{1}$ Amongst sarcomas, lipoid variety is rarest. Vulval lipoid tumours are mostly benign (98\%) with only $2 \%$ being malignant. ${ }^{2}$ A myxoid liposarcoma is often misdiagnosed due to its rarity, location, and similarity to benign lipoma. ${ }^{2,3}$ Asymptomatic presentation is commonest.

Histopathology (HPE) suffices for diagnosis; immunohistochemistry (IHC) is not necessary to distinguish it from other myxoid lesions of vulva. ${ }^{4}$ Surgical resection is the treatment of choice with adjuvant radiotherapy needed in only selected cases. ${ }^{1-3}$ Role of chemotherapy is unproven, but a lifelong follow up is must. ${ }^{3}$ Till now, only 20 such cases have been reported in literature. ${ }^{1-3}$

\section{CASE REPORT}

We present an extremely rare case of a 30 years old unmarried nulliparous woman presenting to our OPD in March 2010 with chief complaint of a giant vulval mass since 2 years causing great difficulty while walking due to its enormous size. The mass was painless, gradually increasing in size from peanut to its present size. Family history was insignificant. General physical and systemic examinations were normal. Local examination revealed a large circumscribed vulval mass of approx $30 \times 20 \mathrm{~cm}$ and weighing nearly $6 \mathrm{~kg}$ which was non tender, firm to cystic in consistency, with the base arising from the right labium majus and having 4 to 5 actively discharging sinuses on the dependent area (Figure 1A-C). The mass was free from the underlying pubic bone with no signs of inflammation and non enlarged inguinal lymph nodes. We kept the differential diagnosis of benign lipoma (due to its rubbery consistency) and elephantiasis of vulva (as she belonged to the endemic area with no other features suggestive of 
malignancy). Her routine laboratory investigations were normal. Ultrasonography (USG) revealed it to be a cystic mass with minimal vascularity probably arising from the vulva. Pelvic contrast magnetic resonance imaging (MRI) showed fatty areas suggesting lipoma. Whole body Positron Emission Tomography-Computed Tomography (PET-CT) scan was negative for distant metastasis. USG guided core biopsy suggested liposarcoma. Patient underwent wide local resection with clear margins (Figure 1D). Post operative recovery was uneventful. Adjuvant radiotherapy of 60 Gy over 30 fractions of 2 Gy each was given. Final histopathology confirmed it as a case of myxoid liposarcoma with variable sized adipocytes and a myxoid stroma with hyperchromatic nuclei, vacuolated lipoblasts and an arborizing delicate capillary network. The patient was followed up for 8 years with no recurrence and no metastasis.

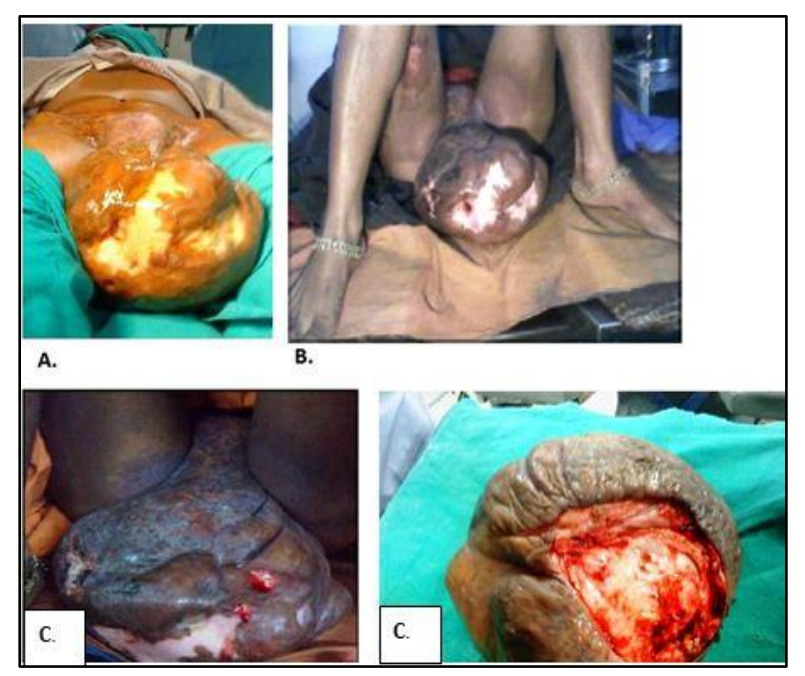

Figure 1: Gigantic myxoid liposarcoma of vulva. (A) Supine view, $(\mathrm{B})$ perineal view, $(\mathrm{C})$ closer view showing discharging sinuses on the dependent portion and (D) excised specimen.

\section{DISCUSSION}

Gynecological malignancies rate second after breast cancer in women. Vulval malignancies are rare $(5 \%$ of total gynaecological malignancies), ranking fourth in position after cervical, uterine and ovarian malignancies. ${ }^{5}$ The variant which abounds the vulval region is squamous cell carcinoma (95\%). Sarcomas represent only 1-3\% of all vulval tumors, with leiomyosarcoma being the most common variety in the bartholins and labia majora (1\%) region followed by epitheloid and rhabdomyosarcomas. ${ }^{1,3,5}$ Lipoid variety of leiomyosarcoma is still rarer, with $98 \%$ being benign and $2 \%$ being malignant. ${ }^{2,3,5}$ Sarcomas, a tumor of adults (20\%) occurs most commonly in the extremities and retroperitoneal region, reaching large sizes and characterized by rapid growth, high metastatic potential, frequent recurrence, aggressive behavior and high mortality rates. ${ }^{1,5}$ The liposarcoma variant being uncommon therefore has not been very well studied. Our literature search shows only around 20 such patients reported till date..$^{1-3,5}$ Its natural history and treatment has also not been standardized. ${ }^{5}$ The myxoid/ round cell liposarcoma variant is most commonly reported in 30-40 years age (range 15-69 years), with the youngest patient reported to be a 15 year old girl with poorly differentiated round cell variant, who died due to recurrence (Brooks et al). ${ }^{1,2,5,6}$ Mean size reported till now is $6.2 \mathrm{~cm}$ (range 1.5$16 \mathrm{~cm}$ ), but our case had a gigantic size of approximately $30 \times 20 \mathrm{~cm} .{ }^{2,5}$ Knowing the histological type is important as it is the main determinant of prognosis apart from primary location and resection margin status. ${ }^{1-3}$ Five commonly described histological subtypes are: well differentiated liposarcoma (WDLS), dedifferentiated liposarcoma (DLS), myxoid cell liposarcoma (MLS), round cell liposarcoma (RCLS), and pleomorphic liposarcoma (PELS). Of these varieties, WDLS (40-45\%) and MLS are the commonest with the best prognosis as they do not metastasize and have an indolent low grade progression. ${ }^{1-}$ 3 Myxoid liposarcoma is a low grade variety lesion growing as a gelatinous mass. ${ }^{2} \mathrm{MRI}$ is the investigation of choice for its diagnosis though IHC has been reported in literature by various authors as being useful. ${ }^{3}$ The management mainly rests on complete surgical resection with or without adjuvant radiotherapy based on case characteristics and resection margin status. ${ }^{1}$ We gave radiotherapy as we were skeptical about recurrence due to large size of tumor as it provides the best guard against local recurrence. ${ }^{1}$ All histological subtypes are notoriously known to recur (36-72\%), hence a thorough follow up throughout life is suggested along with the caution of keeping in the differential list whenever a case of vulval mass is encountered, as it is often misinterpreted due to its benign presenting clinical and radiological features, as was in our case. . $^{1,3,7}$

Other vulval malignant myxoid soft tissues tumors are angiomyxomas, myxoid dermatofibrosarcoma protuberans, botryoid embryonal rhabdomyosarcoma and myxoid leiomyosarcoma.?

Chemotherapy has no role in most of the histological subtypes, but its role in reduction of relapse and metastasis has been described. ${ }^{1,5}$ A thorough literature search in 2014 on 17 cases of myxoid liposarcoma done by Kwak et al reported 10/17 cases of WDLS, 4/17 of MLS, 2/17 of PELS, 1/17 of unknown subtype; 5 of these 17 cases had recurrence. ${ }^{1}$ Schoolmester in 2015 reported 2 cases, one each of MELS and WDLS, both diagnosed for the first time on IHC. ${ }^{2}$

Nucci et al reported 6 cases in middle aged women, 4 were WDLS, one REMS and one of a spindle cell variety with focally infiltrative margins; all managed by local resection with no recurrence at a maximum follow up of 7 years in $4 / 6$ cases. $^{8}$ A liposarcoma has even been described in a pregnant female. ${ }^{5}$ There is still a dearth of literature and more case series are required before standardization of protocols for its management are made. 


\section{CONCLUSION}

Though rare, myxoid liposarcoma should be kept in differential diagnosis of vulval soft tissue mass. Management includes a combination of surgery and radiation. Excision of lymph nodes is not recommended. Strict prolonged follow up for recurrence or metastasis is mandatory, and any new complaints should be addressed promptly. This is particularly true in the setting of liposarcoma, which may exhibit unusual patterns of spread and recurrence.

Funding: No funding sources

Conflict of interest: None declared

Ethical approval: Not required

\section{REFERENCES}

1. Ju Hyun Kwak, Sun Mi Shin. Unusual bilateral vulvar liposarcoma. Obstet Gynecol Sci. 2014;57(6):549-52.

2. Schoolmester JK, Leifer AJ. Vulvar Myxoid Liposarcoma and well differentiated liposarcoma with molecular cytogenetic confirmation: case reports with review of malignant lipomatous tumours of the vulva. Int J Gynecol Pathol. 2015;34(4):390-5.
3. Redroban.L, Montalvo.N. Vulvar myxoid liposarcoma, an extremely rare diagnosis. Int $\mathrm{J}$ Gynecol Pathol. 2017;0(0).

4. Donnellan R, Moodley M. Vulval myxoid liposarcoma. Int J Gynecol Cancer. 2001;11:321-322.

5. AA Chokoeva, G.Tchernev. Vulvar Sarcomas: short guideline for histopathological recognition and clinical management, Part 2. Int J Immunopathol Pharmacol. 2015;28(2):178-86.

6. Brooks JJ, LiVolsi VA. Liposarcoma presenting on the vulva. Am J Obstet Gynecol. 1987;156:73-75.

7. Wu TC, Tarn JJ. Vulvar myxoid liposarcoma. Taiwan J Obstet Gynecol. 2007;46:293-294.

8. Nucci MR, Fletcher CD. Liposarcoma (atypical lipomatous tumours) of the vulva: a clinicopathologic study of six cases. Int $\mathbf{J}$ Gynecol Pathol. 1998;17(1):17-23.

Cite this article as: Kaur T, Singh RP. A rare case of a gigantic myxoid liposarcoma of vulva: case report. Int J Reprod Contracept Obstet Gynecol 2021;10:1174-6. 